\title{
Symmetry Breaking in the Growth of Two-Dimensional Islands on Si(111)
}

\author{
Konstantin Romanyuk, Vasily Cherepanov, and Bert Voigtländer* \\ Institute of Bio- and Nanosystems (IBN 3), and cni-Center of Nanoelectronic Systems for Information Technology, \\ Research Center Jülich, 52425 Jülich, Germany \\ (Received 6 June 2007; published 20 September 2007)
}

\begin{abstract}
We find that the shape of two-dimensional (2D) Si or Ge islands has a lower symmetry than the threefold symmetry of the underlying $\mathrm{Si}(111)$ substrate if $\mathrm{Bi}$ is used as a surfactant during growth. Arrowshaped or rhomb-shaped 2D islands are observed by scanning tunneling microscopy. This symmetry breaking is explained by a mutual shift between the surface reconstructions present on the substrate and on the islands. Using the kinematic Wulff construction the growth velocities of the steps could be determined from the island shape if the nucleation center has been located by a marker technique.
\end{abstract}

DOI: 10.1103/PhysRevLett.99.126103

The observation of faceted crystal shapes is a fascinating experience because it allows a direct look into the point group symmetry of the crystal. The equilibrium shape of a crystal has the same symmetry as the lattice of the atoms inside the crystal [1]. Also, for growth shapes of crystals, often a close relation between the crystal lattice symmetry and the morphology is found. The shape of epitaxially grown islands usually follows the symmetry of the underlying substrate. On the threefold symmetric $\mathrm{Si}(111)$ substrate epitaxially grown two-dimensional $\mathrm{Si}$ and $\mathrm{Ge}$ islands have triangular shape [2]. Also the tetrahedron-shaped three-dimensional $\mathrm{Ge}$ islands grown on $\mathrm{Si}(111)$ obey the threefold symmetry of the substrate [2]. At the $C_{2 v}$ symmetric $\mathrm{Si}(001)$ surface two-dimensional $\mathrm{Si}$ or $\mathrm{Ge}$ islands and the three-dimensional hut clusters have an elongated shape consistent with the $C_{2 v}$ symmetry [3]. It is well known that surface reconstructions can lower the symmetry of the crystal. For instance, for surface reconstructions induced by atomic layer thick adlayers of In and $\mathrm{Au}$, the formation of linear stripes breaks the threefold symmetry of the underlying $\mathrm{Si}(111)$ surface [4,5]. However, this symmetry lowering due to the reconstruction does not imply a pronounced impact on the island shapes on these surfaces [6].

In this Letter we show that surface reconstruction can lead to the growth of two-dimensional epitaxial islands with a lower symmetry than the substrate. In surfactantmediated $\mathrm{Si}$ and Ge epitaxy on the threefold symmetric $\mathrm{Si}$ (111) substrate we observed the growth of arrow-shaped and rhomb-shaped two-dimensional islands, respectively. Interestingly, these islands break the threefold symmetry of the substrate in spite of the fact that the $(\sqrt{3} \times \sqrt{3}) R 30^{\circ}$ $\mathrm{Bi}$ reconstruction present at the surface has locally the same threefold symmetry $\left(C_{3 v}\right)$ as the $\mathrm{Si}(111)$ substrate. The reason for the symmetry breaking is a mutual shift between the reconstruction on the substrate and on the island, imposed by the diamond crystal structure of the $\mathrm{Si}$ substrate. The shape of the islands can be related to the growth velocities of the step edges via the kinematic Wulff construction resulting in a complete information on
PACS numbers: 68.35.Bs, 68.37.Ef, 68.55.Ac, 68.65.-k

the growth kinetics of the islands shown in a kinematic Wulff plot.

In our experiments we use surfactant-mediated growth $[7,8]$, which means that an additional species floating at the growth front (Bi in our case) modifies epitaxial growth. Prior to growth a saturation coverage of one atomic layer of $\mathrm{Bi}$ is deposited on the clean $\mathrm{Si}(111)-(7 \times 7)$ at $740 \mathrm{~K}$ [9]. Subsequently, a submonolayer amount of $\mathrm{Si}$ or Ge was deposited at growth rates of about 0.02 atomic layers per min and at a temperature of $670 \mathrm{~K}$. A Bi flux of one atomic layer per min was maintained during Ge growth to keep a complete Bi termination. After growth the surface was imaged at room temperature by scanning tunneling microscopy (STM).

Figure 1(a) shows two-dimensional Si islands grown on the $\mathrm{Si}(111)$ surface in Bi mediated epitaxy. Clearly these arrow-shaped islands have a lower symmetry than the threefold symmetry $\left(C_{3 v}\right)$ of the underlying $\mathrm{Si}(111)$ surface. The symmetry of an island is reduced to $C_{s}$ symmetry with only one mirror plane as symmetry operation. The arrow-shaped islands occur in three domains. The crystallographic directions of the steps terminating the islands were determined from comparison to atomically resolved STM images of the clean $\operatorname{Si}(111)-(7 \times 7)$ surface taken

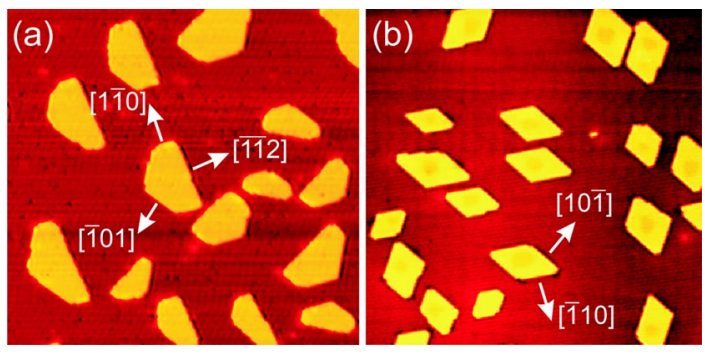

FIG. 1 (color online). (a) STM images of arrow-shaped Si islands on the $\mathrm{Bi}$ terminated $\mathrm{Si}(111)$ substrate. The shape of these islands breaks the threefold symmetry of the substrate surface. (b) Rhomb-shaped $\mathrm{Ge}$ islands on the Bi terminated $\mathrm{Si}(111)$ substrate breaking the threefold symmetry as well. Image sizes (a) $200 \mathrm{~nm}$, (b) $110 \mathrm{~nm}$. 
before deposition of $\mathrm{Bi}$. The $(\sqrt{3} \times \sqrt{3}) R 30^{\circ} \mathrm{Bi}$ reconstruction is present on the substrate as well as on the islands. For the growth of $\mathrm{Ge}$ on the $\mathrm{Bi}$ terminated $\mathrm{Si}(111)$ rhomb-shaped islands [terminated by the $(\sqrt{3} \times$ $\sqrt{3}$ ) Bi reconstruction as well] were observed, also breaking the threefold symmetry of the $\mathrm{Si}(111)$ substrate surface [Fig. 1(b)]. As will be shown later, the symmetry of the rhomb-shaped island is also $C_{s}$.

The observed symmetry breaking is a striking result because the $\mathrm{Bi}(\sqrt{3} \times \sqrt{3})$ reconstructions on both, the island and the substrate, obey the same symmetry as the substrate. The reason for the observed symmetry breaking is a mutual lateral shift between the reconstructions on the substrate and on the islands. Figure 2(a) shows two adjacent diamond structure (111) bilayers: the substrate bilayer and a hexagonal island residing on the substrate. The shape of the island was chosen such that all six experimentally observed step edges of the $\langle 1 \overline{1} 0\rangle$ type (Fig. 1) are included. Without taking the $\mathrm{Bi}$ reconstruction into account, the combined system of island plus the substrate obeys the $C_{3 v}$ symmetry with three mirror planes (the combined system without reconstruction is still part of the bulk crystal). This means also that the step edges marked as 1 , 2, and 3 in Fig. 2(a) have the same structure. Now we include the Bi reconstruction which is indicated by colored

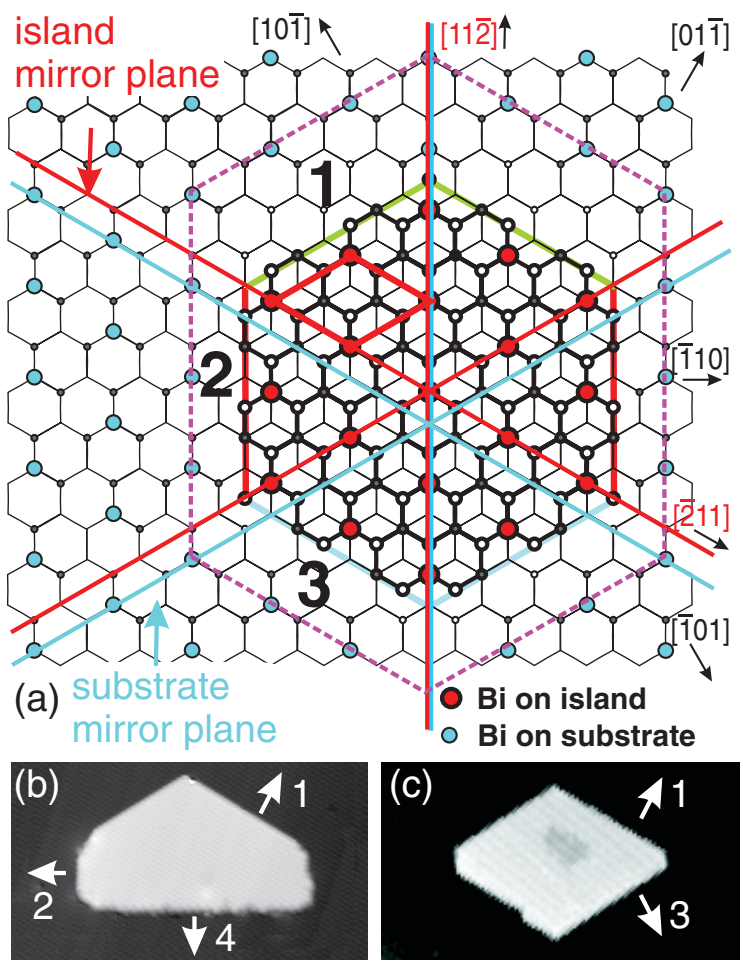

FIG. 2 (color). (a) Schematic of the two level system of the substrate and a two-dimensional island. While a threefold symmetric $(\sqrt{3} \times \sqrt{3})$ Bi reconstruction is present on each individual level, the $C_{3 v}$ symmetry is broken for the combined system and is reduced to a $C_{s}$ symmetry. (b) and (c) STM images of an arrow- and a rhomb-shaped shaped island, respectively. circles in Fig. 2(a) (one circle corresponds to a Bi trimer). Because of the reconstruction two of the mirror planes of the substrate reconstruction (blue lines) are not coincident with the mirror planes of the island reconstruction (red lines). While the two sets of substrate and island mirror planes run along the same directions the common point of the $C_{3}$ point symmetry operations is shifted between substrate and island by the vector $a_{0} / \sqrt{3}\left[\begin{array}{lll}1 & 1 & 2\end{array}\right]$. Therefore, the symmetry of the combined system of island and substrate is lowered. Only the mirror plane running along [112̄] is a symmetry element of the combined system. Because of the shift of the common point of the other two mirror planes between island and substrate reconstruction these symmetry operations are no longer point symmetry operations of the combined system.

The lateral shift between the reconstructions on the island and the substrate is induced by a lateral shift between two adjacent (111) bilayers of the diamond lattice of the same value $a_{0} / \sqrt{3}\left[\begin{array}{lll}1 & 1 & 2\end{array}\right]$ [compare Fig. 2(a)]. However, for the unreconstructed system this shift (which is a property of the bulk crystal structure) does not break the $C_{3 v}$ symmetry. This mutual lateral shift between both bilayers also propagates to the $\mathrm{Bi}(\sqrt{3} \times \sqrt{3})$ reconstruction layers in both levels. However, on the reconstructed surface the threefold symmetry of the underlying substrate is broken for the combined system because the common points of the point symmetry operations of both subsystems are shifted relative to each other due to the larger unit cell of the reconstructed surface. Generally, for two systems and the corresponding two sets of point group operations $A$ and $B$ the set of point group operations of the combined system $D$ is the intersection $D=A \cap B$ [10]. This means that the only symmetry plane of the combined system is the [112] mirror plane and the resulting symmetry group of the combined system is $C_{s}$ [11].

The reduced symmetry of the combined system of reconstructed surface and island has important consequences for the shape of the islands. The neighboring steps 1,2, and 3 , respectively, are not connected by a symmetry operation as the $C_{3 v}$ symmetry is broken for the combined system. Thus, these steps are all different. These differences in the structure of the step edges lead to different step speeds. The different step speeds in turn determine the island shapes, as we will show. While we do not know the detailed atomic step structure, the difference between the steps can also be inferred from the mutual shift of the reconstructions of the substrate and the island [Fig. 2(a)]. For instance, at step 2 the Bi trimers on the substrate and the island (blue and red circles, respectively) have a much smaller mutual distance than for the step 1. These differences have important consequences on the $\mathrm{Bi}$ termination of the step edges. For instance, one might speculate that at step 2 a Bi termination of the step edge is not possible because the $\mathrm{Bi}$ on the lower terrace blocks the possible bonding sites, while for step 1 $\mathrm{Bi}$ passivation is easily possible. These different structures of the step edges (imposed by the symmetry) have impor- 
tant consequences on the growth properties of the islands. A Bi passivated step edge is expected to have a completely different incorporation kinetics than an unpassivated step edge [12]. For an initially arbitrary shape of an island, easy incorporation of material leads to a high step velocity (rate of advancement of the step edge perpendicular to the step edge). These step edges with a higher incorporation rate grow faster in the direction perpendicular to the step edge and can in some cases even finally disappear being overgrown by the nearest steps with lower growth rate. The formation of the observed rhomb-shaped islands is explained by a slow growth velocity of steps 1 and 3 which leads to a disappearance of the fast growing step 2. This is a consequence of the more general kinematic Wulff construction [13]. The original Wulff construction (2D version) relates the equilibrium crystal shape to the step energy; the kinematic Wulff construction relates the crystal growth shape to the step velocities. The kinematic Wulff theorem (2D version) states that the shortest distances from the nucleation center to the step edge, $h_{i}$ [inset in Fig. 3(a)] are related to the step velocities $v_{i}$ by

$$
\frac{v_{1}}{h_{1}}=\frac{v_{2}}{h_{2}}=\frac{v_{3}}{h_{3}}=\ldots=\text { const. }
$$

A prerequisite in the model underlying the kinematic Wulff construction is that the velocity of growth depends only on the surface orientation. This is true for a growth regime in which the incorporation into the steps is attachment limited and the adatom concentration on the surface is approximately constant due to a large incorporation barrier. Since strong step edge passivation was found in

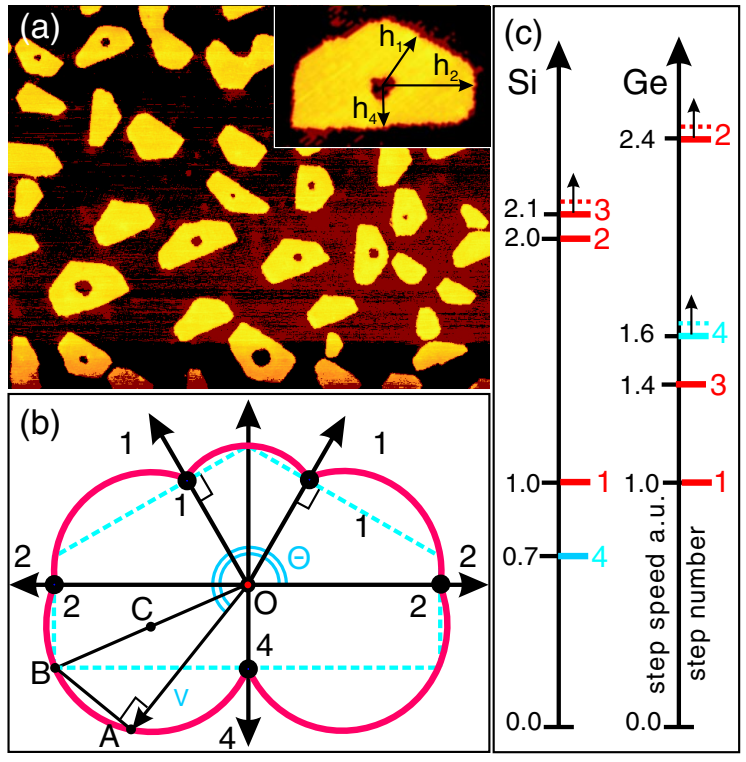

FIG. 3 (color online). (a) Using a marker technique the nucleation centers of the arrow-shaped islands are marked by holes. From the measured distances to the step edges the step velocities can be determined. Image size $400 \mathrm{~nm}$. (b) Minimal kinematic Wulff Plot of arrow-shaped islands. (c) Step velocities for Si and Ge islands.
Bi surfactant-mediated epitaxy of Ge [12], our experiments are performed under conditions in which this prerequisite for the kinematic Wulff theorem is fulfilled. Another requirement for the validity of the kinematic Wulff theorem is that the island shape is a growth shape and not influenced by the equilibrium shape due to the low growth rate used. STM experiments performed during epitaxial growth [2] of Si showed that the growth shape develops already for much lower growth rates than used here.

In order to obtain the step velocities from Eq. (1) the nucleation point of the island has to be known, from which the distances $h_{i}$ are measured. Without this information often only a limited number of relations among the step velocities can be obtained. If the nucleation point of the island is known, the complete knowledge of the velocities of the observed steps results from the shape of the island. Therefore, we used a marker technique to determine the position of the nucleation center inside the arrow-shaped $\mathrm{Si}$ islands. Initially we deposited small $\mathrm{Ge}$ islands which served as markers of the nucleation centers. Subsequently, larger $\mathrm{Si}$ islands were grown around the marked nucleation centers. Actually, due to an effect induced by strain (which will not be discussed here in detail) the Ge diffuses away from its initial position (leading to a small $<5 \% \mathrm{Ge}$ alloying of the $\mathrm{Si}$ islands) and the nucleation center of the island is marked by a small hole of one atomic layer height. With the nucleation point known, the distances $h_{i}$ to the island edges can be easily measured [inset in Fig. 3(a)] and the relative step velocities can be determined from Eq. (1). Choosing $v_{1}=1$ results in: $v_{2}=$ $2.0 \pm 0.1$ and $v_{4}=0.7 \pm 0.1$. These measured step velocities can be plotted in a kinematic Wulff plot [polar diagram of the step velocities $v(\theta)$ ] for the arrow-shaped islands. The measured values correspond to the five black dots in Fig. 3(b).

If the growth shape would be smooth without straight step segments, the inverse Wulff construction could be used to obtain the complete polar diagram of the step velocities from the measured island shape [13]. Because of the presence of the straight step edges (facets) in the island shape the inverse Wulff construction is not possible. However, one can learn more than just the determination of the five measured points on the kinematic Wulff plot. In the following it will be shown that the step velocities have to lie outside the red contour in Fig. 3(b) which we call the "minimal" kinematic Wulff plot. According to the kinematic Wulff construction a line perpendicular to the radius vector is constructed at each point where the radius vector crosses the polar plot of the step velocity. The crystal shape is formed from the inner envelope of these perpendicular lines for all radius vectors. The inverse Wulff construction which constructs the step velocities from the crystal shape is impossible for step velocities inside the minimal kinematic Wulff plot. Therefore, the step velocities have to lie outside the minimal kinematic Wulff plot. The geometric construction rule for the border of the minimal kinematic Wulff plot is given in the following. For a certain polar 
angle $\theta$ the closest point for which the perpendicular line does not cut the crystal is point $A$ in Fig. 3(b). This perpendicular line just reaches the island corner in $B$. If we now consider the rectangular triangle $O A B$, the converse Thales' theorem states that a right triangle's hypotenuse is a diameter of its circumcircle. This means that all points $B$ lie on a circle around point $C$. With $C$ lying in the middle between the nucleation center and the respective island corner.

The measured step velocities for $\mathrm{Si}$ islands can also be displayed as a "spectrum" shown in Fig. 3(c). The (relative) velocities for steps 1,2 , and 4 have a defined value, while for step 3 the step velocity has to be larger than the value given from the minimal kinematic Wulff plot. An interesting result from the determination of the step velocities is that the velocities of the different kinds of $\langle 1 \overline{1} 0\rangle$ type steps [marked in red in Fig. 3(c)] differ by more than a factor of 2. This is the case in spite of the fact that the local step structure, without taking the reconstruction into account, is the same for all $\langle 1 \overline{1} 0\rangle$ type steps (i.e. steps 1,2 , and 3). The difference between these steps is their shift with respect to the substrate reconstruction and the related differences of the Bi termination. Our results show that these effects have a stronger influence on the step velocities than the difference between $\langle 1 \overline{1} 0\rangle$ and the $\langle\overline{1} \overline{1} 2\rangle$-type steps (step 4 is a $\langle\overline{1} \overline{1} 2\rangle$-type step marked in blue) which have a genuine different structure even without taking the substrate reconstruction into account.

Coming back to the rhomb-shaped islands, also here a marker technique can be used to learn more about the kinematic properties of growth expressed by the step velocities. The STM image in Fig. 2(c) shows an island for which an initial small Si island marks the nucleation position. Si and Ge can be distinguished in Bi mediated epitaxy by an apparent height in the STM images. Ge areas are imaged $\sim 1 \AA$ higher than $\mathrm{Si}$ areas [14]. It is clearly visible that the nucleation center is not located in the center of the rhomb [Fig. 2(c)]. This proves experimentally that the growth velocities of steps 1 and 3 are different which was already concluded from symmetry considerations. A detailed analysis shows that the average step velocity of step 3 is $1.4 \pm 0.1$ times larger than that of step 1 . While the apparent growth shape of the island is rhomb-shaped, the true shape has $C_{s}$ symmetry. Symmetry considerations and the marker technique prove that steps 1 and 3 are different on the atomic scale leading to different growth velocities. Because of geometric constraints the length of both steps is the same leading to a rhomb shape of the islands.

It is an intriguing fact that in spite of the similarity of Si and Ge the epitaxial islands have so different shapes. These different shapes are related, via the step velocities, to different step structures. Since $\mathrm{Si}$ and Ge have the same crystal structure one would not expect much different step structures. However, the $\mathrm{Bi}$ termination at the step edges can be very different for $\mathrm{Si}$ and Ge. For instance in an earlier experiment we found that $\mathrm{Si}$ step edges are less $\mathrm{Bi}$ passivated than Ge step edges [12]. Because of the different bonding of $\mathrm{Bi}$ and strain effects at $\mathrm{Si}$ and $\mathrm{Ge}$ steps a different $\mathrm{Bi}$ passivation, or (partial) depassivation may occur at some step edges. We speculate that a largely different step structure due to different Bi passivation of the steps can lead to substantially modified step velocities for $\mathrm{Si}$ and $\mathrm{Ge}$ and can explain the observed different $\mathrm{Si}$ and Ge island shapes.

Our findings are not limited to a specific system. Generally one can conclude that on reconstructed surfaces the symmetry of the combined system of epitaxially grown islands and substrate can break the symmetry of the original surface. This broken symmetry has consequences on the step structure which in turn influences the growth rates and in turn also the island shapes. This effect could be used to fabricate 2D nanostructures directed along one specific direction on a substrate of higher symmetry. If one of the step velocities would be much larger than the others the island shape would develop a pronounced oblong shape. In order to grow elongated nanostructures (surfactant induced) reconstructions which generate a large anisotropy in step velocities have to be found.

We gratefully acknowledge critical reading of the manuscript by H. P. Bonzel and S. N. Filimonov. K. R. acknowledges the support of the NANOCAGE Marie Curie early stage training network.

*Corresponding author. b.voigtlaender@fz-juelich.de

[1] A.A. Chernov, Modern Crystallography 3-Crystal Growth (Springer, Berlin, 1984).

[2] B. Voigtländer, Surf. Sci. Rep. 43, 127 (2001).

[3] Y.-W. Mo, D. E. Savage, B. S. Swartzentruber, and M. G. Lagally, Phys. Rev. Lett. 65, 1020 (1990).

[4] J. Nogami, Sang-il Park, and C. F. Quate, Phys. Rev. B 36, 6221 (1987).

[5] A. A. Baski, J. Nogami, and C. F. Quate, Phys. Rev. B 41, 10247 (1990).

[6] H. Minoda, Y. Tanishiro, N. Yamamoto, and K. Yagi, Surf. Rev. Lett. 2, 1 (1995).

[7] M. Copel, M. C. Reuter, E. Kaxiras, and R. M. Tromp, Phys. Rev. Lett. 63, 632 (1989).

[8] M. Horn-von Hoegen, Appl. Phys. A 59, 503 (1994).

[9] R. Shioda, A. Kawazu, A. A. Baski, C. F. Quate, and J. Nogami, Phys. Rev. B 48, 4895 (1993).

[10] F. Bechstedt, Principles of Surface Physics (Springer, Heidelberg, 2003).

[11] The occurrence of three different domains of arrowshaped or rhomb-shaped islands observed in the STM experiment (Fig. 1) can be explained by three $\left(120^{\circ}\right.$ rotated) domains of the $(\sqrt{3} \times \sqrt{3})$ reconstruction on the islands.

[12] V. Cherepanov, S. N. Filimonov, J. Myslivecek, and B. Voigtländer, Phys. Rev. B 70, 085401 (2004).

[13] A. Pimpinelli and J. Villain, Physics of Crystal Growth (Cambridge University Press, Cambridge, England, 1998).

[14] M. Kawamura, N. Paul, V. Cherepanov, and B. Voigtländer, Phys. Rev. Lett. 91, 096102 (2003). 